\title{
Kinetics of virus entry by endocytosis
}

\author{
Vladimir P. Zhdanov* \\ Department of Applied Physics, Chalmers University of Technology, S-41296 Göteborg, Sweden \\ and Boreskov Institute of Catalysis, Russian Academy of Sciences, Novosibirsk 630090, Russia
}

(Received 20 December 2014; published 28 April 2015)

\begin{abstract}
Entry of virions into the host cells is either endocytotic or fusogenic. In both cases, it occurs via reversible formation of numerous relatively weak bonds resulting in wrapping of a virion by the host membrane with subsequent membrane rupture or scission. The corresponding kinetic models are customarily focused on the formation of bonds and do not pay attention to the energetics of the whole process, which is crucially dependent, especially in the case of endocytosis, on deformation of actin filaments forming the cytoskeleton of the host cell. The kinetic model of endocytosis, proposed by the author, takes this factor into account and shows that the whole process can be divided into a rapid initial transient stage and a long steady-state stage. The entry occurs during the latter stage and can be described as a first-order reaction. Depending on the details of the dependence of the grand canonical potential on the number of bonds, the entry can be limited either by the interplay of bond formation and membrane rupture (or scission) or by reaching a maximum of this potential.
\end{abstract}

DOI: 10.1103/PhysRevE.91.042715

PACS number(s): 87.19.xd, 87.16.D-, 87.16.Ln

\section{INTRODUCTION}

Viruses are biological nanoparticles with diameter ranging typically from 60 to $150 \mathrm{~nm}[1,2]$. Their DNA or RNA genome is protected by a protein capsid and sometimes also by a lipid membrane envelope. The viral replication cycle includes virion attachment to a host-cell lipid membrane, penetration, uncoating and release of genome, genome replication, viral protein synthesis, capsid assembly, and escape from the host [1]. The understanding of the mechanistic details of these steps is obviously important from the perspective of various biomedical applications. In addition, this area is of considerable interest in the context of statistical and soft matter physics. At the current state of the art, the kinetics models describing various aspects of the viral replication cycle (see, e.g., [3,4] and references therein) are inevitably coarse grained. The most detailed models have been proposed for capsid assembly (see, e.g., [5], recent review [6], and references therein).

Herein, we focus on the kinetics of virus entry into the host cells (Fig. 1). This step is either endocytotic, with wrapping of a virion by the host membrane and subsequent formation of a vesicle covering a virion, or fusogenic, with fusion of the virus membrane envelope with the cell membrane (the corresponding experimental studies are reviewed in [2,7]). In both cases, it occurs via formation of a large number of relatively weak bonds between viral proteins, referred to below as ligands, and appropriate host cell receptors (specific membrane proteins, lipids, or glycans [8]).

Endocytosis is the most common pathway of entry of both naked and membrane-enveloped viruses, because in principle it does not require any specific virus counterparts except the viral proteins mediating this process. Scrutinizing the details of endocytosis, one can distinguish different channels of this process $[2,7,9]$. One of the most common channels includes formation of a clathrin (cytoplasmic protein) coat on the cytoplasmic leaflet of the plasma membrane [2]. Another channel, macropinocytosis, is induced by growth factors and often involves the formation of membrane ruffling and large

\footnotetext{
*zhdanov@ catalysis.ru
}

vacuoles [7]. Caveolar raft-mediated endocytosis depends on cholesterol and a complex signaling pathway involving tyrosine kinases and phosphatases [2]. There are also other still poorly characterized channels [2,7,9]. At present, virus entry can be studied at the level of single virions [10]. The understanding of the mechanistic details of this process and the physics behind it is, however, still limited.

The available kinetic models of virus entry can be divided into two related groups. The first one includes the coarsegrained models operating with the populations of virions outside cells, at the cell membrane, and inside cells (see, e.g., [11] and references therein). In such models, the virus-cell interaction is described at the level of the rate constants of virion attachment to, detachment from, and penetration of the membrane. The models of the second category are focused on the virus entry itself with emphasis on the formation of the ligand-receptor bonds [12-16] or, more specifically, on the interplay of virion diffusion and bond formation [12], competition between fusion and endocytosis [13], diffusion of receptors [14], membrane lipid segregation [15], virion detachment [4], and inhibition of virion attachment [16]. In all these models, the constants and/or rate constants describing the ligand-receptor interaction are considered to be parameters, and the energetics of the virion-cell contact is not taken explicitly into account. The analysis of the energetics has been performed [17,18] (see also related studies focused on nanoparticles [19]) but without paying attention to the kinetics.

During endocytosis, the deformation of the host-cell membrane and cytoskeleton below is appreciable, and the corresponding changes in the energy are appreciable as well $[17,18]$. This circumstance motivates explicit inclusion of the energetics into the kinetic models describing this process. Below, we show how it can be done and what can be learned following this way.

\section{PHENOMENOLOGY}

\section{A. In terms of the engulfment depth}

The energy of interaction of a virion with the host-cell membrane is usually calculated $[17,18]$ as a function of the 


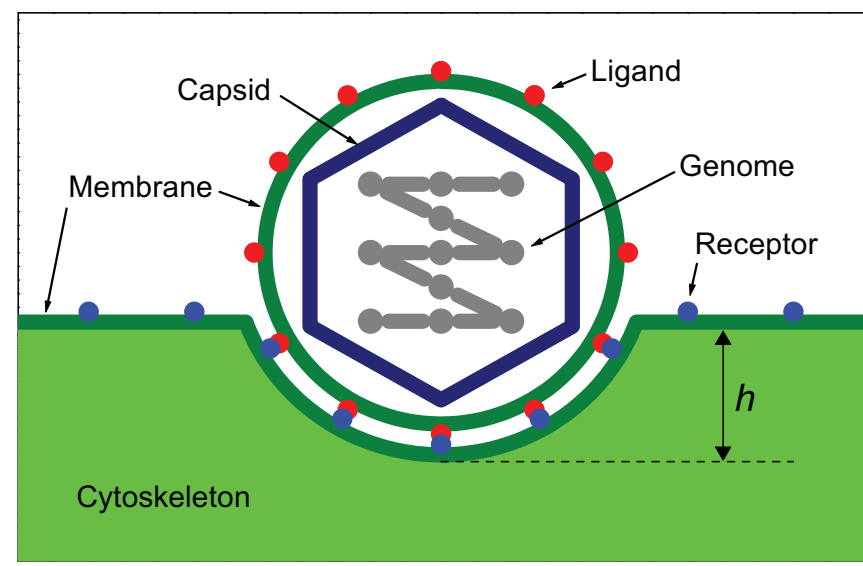

FIG. 1. (Color online) Scheme of virion engulfment into the host cell due to the ligand-receptor interaction (adopted from [18]).

engulfment depth $h$ (Fig. 1). For a spherically shaped virion (with radius $R$ ), this energy can be represented at the generic level as [18]

$$
\begin{gathered}
E(h)=E_{\mathrm{lr}}(h)+E_{\mathrm{b}}(h)+E_{\mathrm{c}}(h), \text { with } \\
E_{\mathrm{lr}}(h)=-2 \pi \alpha h R, \\
E_{\mathrm{b}}(h)=4 \pi \kappa h / R, \\
E_{\mathrm{c}}(h)= \begin{cases}0 & \text { for } h<h_{\circ}, \\
\beta\left(h-h_{\circ}\right)^{4} & \text { for } h>h_{\circ},\end{cases}
\end{gathered}
$$

where $E_{\mathrm{lr}}$ is the attractive ligand-receptor interaction $(2 \pi h R$ is the contact area, and $\alpha$ is the interaction energy per unit area), $E_{\mathrm{b}}$ is the membrane bending energy ( $\kappa$ is the corresponding modulus), and $E_{\mathrm{c}}$ is the deformation energy of cytoskeleton or, more specifically, of actin filaments ( $\beta$ is the corresponding coefficient, and $h_{\circ}$ is the engulfment depth corresponding to the beginning of deformation of actin filaments).

Expression (2) for $E_{\mathrm{lr}}$ is phenomenological and does not describe explicitly the statistics of formation of the ligandreceptor bonds $[17,18]$ [cf. Eq. (7) below]. The proportionality of $E_{\mathrm{lr}}$ to the contact area implies that the number of ligand-receptor pairs is proportional to this area. The lateral interaction between these pairs is considered to be weak (compared to $E_{\mathrm{lr}}$ ) and neglected. [In principle, the latter interaction can easily be taken into account in the mean-field or quasichemical approximations [20] or by using Monte Carlo simulations [4]. To keep our presentation compact, we do not introduce the corresponding term into Eq. (2).]

Expression (3) represents the contribution of the contact area into the membrane bending energy [17,18]. The contribution of the membrane deformation near the rim (at the boundary of this area) into the energy is neglected, because quantitatively its role is minor [see, e.g., the results of detailed numerical calculations shown in Figs. 3 and 4 in [19](d)], and its addition does not change the analysis of the penetration process and the main conclusions. Mechanistically, the role of the rim appears to be crucial at the late stage of endocytosis when the membrane near the rim can be viewed as a narrow neck. The membrane curvature in this region becomes appreciable, and one could expect that the corresponding bending energy would be appreciable as well. In the phenomenological treatment of the membrane deformation with the size-independent bending modulus, this is, however, not the case, because the membrane deformation for the neck is nearly catenoidal, which results in vanishing mean curvature and small corresponding bending energy [see, e.g., [19] $(\mathrm{d}, \mathrm{e})]$. On the other hand, the size of virions is small and it is expected to result in deviations from the conventional phenomenology [21]. For this reason, the curvature-dependent membrane strain related to appreciable bending near the neck is likely to facilitate the membrane rupture or scission at this stage (in analogy with rupture of vesicles on solid supports [22]; concerning the role of membrane curvature in various membrane processes, see also, e.g., [23]).

The virion-induced deformation of the cytoskeleton is often described using the conventional continuum theory of elasticity without specification of its structure [17]. The size of virions is, however, smaller than or comparable to the length scale characterizing the cytoskeleton heterogeneity (reviewed in [24]). On this length scale, the cytoskeleton can be viewed as a actin filament network spanning the cells interior as schematically shown in Fig. 2. This network is stabilized by cross-linking proteins (the corresponding models, e.g. [25], are reviewed in [26]). During diffusion on the cell membrane, a virion can find regions where the distance between actin filaments is comparable to its size and the energetic barrier for endocytosis is reduced. In such favorable

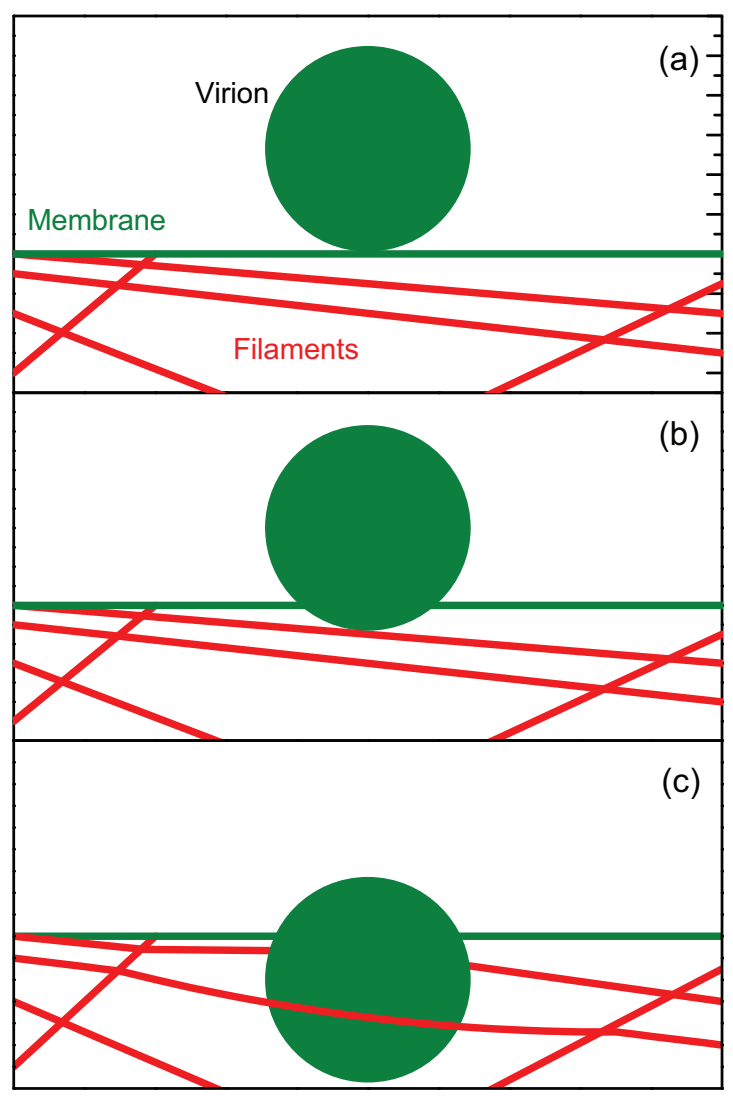

FIG. 2. (Color online) Scheme of virion engulfment into the host cell with emphasis on the role of deformation of actin filaments forming the cytoskeleton: (a) just before the engulfment (at $h=0$ or $n=1)$, (b) in the beginning of deformation of actin filaments ( $h=h$ 。 or $n=h_{\circ}$ ), and (c) at the maximal deformation $\left(h=h_{*}\right.$ or $\left.n=n_{*}\right)$. 
cases, the underlying actin mesh impedes endocytosis only when $h$ becomes comparable to $R$, i.e., at $h>h_{\circ}$. The corresponding filament deformation energy can be represented as a sum of the stretching and bending energies [18] (note that the elasticity theory should in this case be used at the level of filaments). With increasing $h$, the stretching rapidly becomes more important (see the analysis in [18]). Employing expression (4), we take only the stretching energy into account. (Note that the filaments are deformed by a virion in the directions perpendicular to their axes. For this reason, the scale of the filament strain is $\left(h-h_{\circ}\right)^{2} / l_{\mathrm{f}}^{2}$, where $l_{\mathrm{f}}$ is the filament length. The stretching energy is proportional to the square of the strain, i.e., to $\left(h-h_{\circ}\right)^{4}[18]$.)

The applicability of expression (4) may be limited, because during virion engulfment the filament stretching energy first increases but then, with appreciable engulfment [Fig. 2(c)], the filaments may start to contact the upper part of a virion, and the filament deformation will start to decrease. In other words, this means that after reaching the critical engulfment length $h_{*}$, the stretching energy may start to decrease. This situation can be described by replacing expression (4) at $h>h_{*}$ by

$$
E_{\mathrm{c}}(h)=\beta\left(2 h_{*}-h-h_{\circ}\right)^{4} .
$$

The latter expression is of the same shape as (4) except that it decreases with increasing $h$. [Note that at $h \geqslant h_{*}$ one can write $h=h_{*}+\Delta h$, where $\Delta h \equiv h-h_{*} \geqslant 0$; accordingly Eq. (5) can be rewritten as $E_{\mathrm{c}}(h)=\beta\left(h_{*}-h_{\circ}-\Delta h\right)^{4}$, where $h_{*}-$ $h_{\circ}>0$. For $\Delta h \geqslant 0$, this expression is maximal at $\Delta h=0$ and then decreases with increasing $\Delta h$.]

\section{B. In terms of the number of bonds}

If we are interested in the kinetics of endocytosis, the suitable variable is the number of ligand-receptor bonds $n$, between a virion and the host-cell membrane. To relate $n$ with $h$, we introduce the maximum number of bonds at given $h$,

$$
N=2 \pi h R / s
$$

where $s$ is the contact area per receptor. With these variables, the grand potential for a virion (this potential is convenient because the number of bonds is not constant) can be set as

$$
\begin{aligned}
\Omega(n, N)= & k_{\mathrm{B}} T[n \ln (n / N)+(N-n) \ln (1-n / N)] \\
& -\epsilon n-\mu n+8 \pi \kappa N / m+E_{\mathrm{c}}(N) .
\end{aligned}
$$

The first and second terms at the right-hand side of this expression represent the bond entropy and energy $(\epsilon>0$ is the energy of a single bond; the entropy is calculated in the conventional lattice-gas approximation taking the saturation of bonds into account [20]).

The third term, accounting that the system is open with respect to receptors, contains the chemical potential of receptors,

$$
\mu=k_{\mathrm{B}} T \ln (a c),
$$

where $c$ is the two-dimensional receptor concentration, and $a$ is the area comparable with that of the receptor cross section. This expression for $\mu$ is correct because in vivo, the number of specific receptors is much smaller than the number of lipid molecules in the membrane. In particular, the physiological cellular receptor density is considered to be $\sim 10^{12} \mathrm{~mol} / \mathrm{cm}^{2}[12](\mathrm{b})$, or, in other words, about $0.5 \%$ of the number of lipid molecules in the membrane leaflet. For example, the total ganglioside content can reach up to $1 \%-2 \%[12](\mathrm{d})$.

The fourth term in (7) is the bending energy (3) rewritten in terms of $N$. The last fifth term is the cytoskeleton energy [(4) and (5)] also rewritten in terms of $N$,

$$
E_{\mathrm{c}}(N)=B \begin{cases}0 & \text { for } \quad N<N_{\circ}, \\ \left(N-N_{\circ}\right)^{4} & \text { for } \quad N_{\circ} \leqslant N \leqslant N_{*}, \\ \left(2 N_{*}-N-N_{\circ}\right)^{4} & \text { for } \quad N_{*} \leqslant N \leqslant m,\end{cases}
$$

where $B$ is the parameter corresponding to $\beta, N_{\circ}$ and $N_{*}$ are the parameters corresponding $h_{\circ}$ and $h_{*}$, and $m=4 \pi R^{2} / s$ is the maximum number of bonds at full engulfment.

As already noticed, the concentration of receptors in the host-cell membrane is usually not high, and the attachment and detachment of receptors to a virion is expected to be slower than the local relaxation of the membrane around a virion. Mathematically, this means that potential (7) can be minimized with respect to $N$ at given $n$, i.e., $\partial \Omega / \partial N=0$. This procedure yields

$$
-k_{\mathrm{B}} T \ln (1-n / N)=8 \pi \kappa / m+\frac{d E_{\mathrm{c}}(N)}{d N} .
$$

With realistic values of the parameters (e.g., Table I in [18]), the right-hand side of this equation is appreciably larger than $k_{\mathrm{B}} T$ (taking, e.g., into account that $\kappa \simeq 25 k_{\mathrm{B}} T$ [27] and using $m=$ 60 , one can obtain that the first term alone is about $10 k_{\mathrm{B}} T$ ), and accordingly $N$ is close to $n$, i.e., $n \simeq N=2 \pi h R / s$. In this case, the entropic contribution in (7) can be neglected, $N$ can be replaced by $n$, and $N_{*}$ can be identified with $n_{*}$. With these simplifications, potential (7) can be represented as

$$
\Omega(n)=-A n+B f_{\mathrm{c}}(n),
$$

where $A=\epsilon+\mu-8 \pi \kappa / m$, and

$$
f_{\mathrm{c}}(n)= \begin{cases}0 & \text { for } n<n_{\circ}, \\ \left(n-n_{\circ}\right)^{4} & \text { for } \quad n_{\circ} \leqslant n \leqslant n_{*}, \\ \left(2 n_{*}-n-n_{\circ}\right)^{4} & \text { for } \quad n_{*} \leqslant n \leqslant m .\end{cases}
$$

Expression (10) is compact and convenient in order to form a basis for the analysis of the kinetics of endocytosis. In fact, $n$ can be considered as a coordinate for endocytosis, and this process can be viewed as diffusion occurring along this coordinate and governed by potential (10). The membrane rupture or scission resulting in completion of the process can be considered as reaction taking place provided $n$ is larger than the corresponding critical value, $n_{\mathrm{cr}}\left(n_{\mathrm{cr}}\right.$ is close to $\left.m\right)$, i.e., $n \geqslant n_{\text {cr. }}$. Following this line, a few possible qualitatively different scenarios of endocytosis can be distinguished. First of all, the kinetics depend on whether the deformation energy of cytoskeleton is fully repulsive [Figs. 3(a) and 3(b)], i.e., represented by (4) up to $h=2 R$ [or by the corresponding term in (10) up to $n=m$ ], or first increases at $n \leqslant n_{*}$ and then decreases at $n>n_{*}$ [Fig. 3(c)]. In the former case, after reaching a minimum, $\Omega(n)$ increases with increasing $n$ up to $n=m$, i.e., the states with $n \geqslant n_{\text {cr }}$ becomes statistically less favorable with increasing $n$. In the latter case, after reaching a minimum, $\Omega(n)$ first increases, then reaches a maximum at 


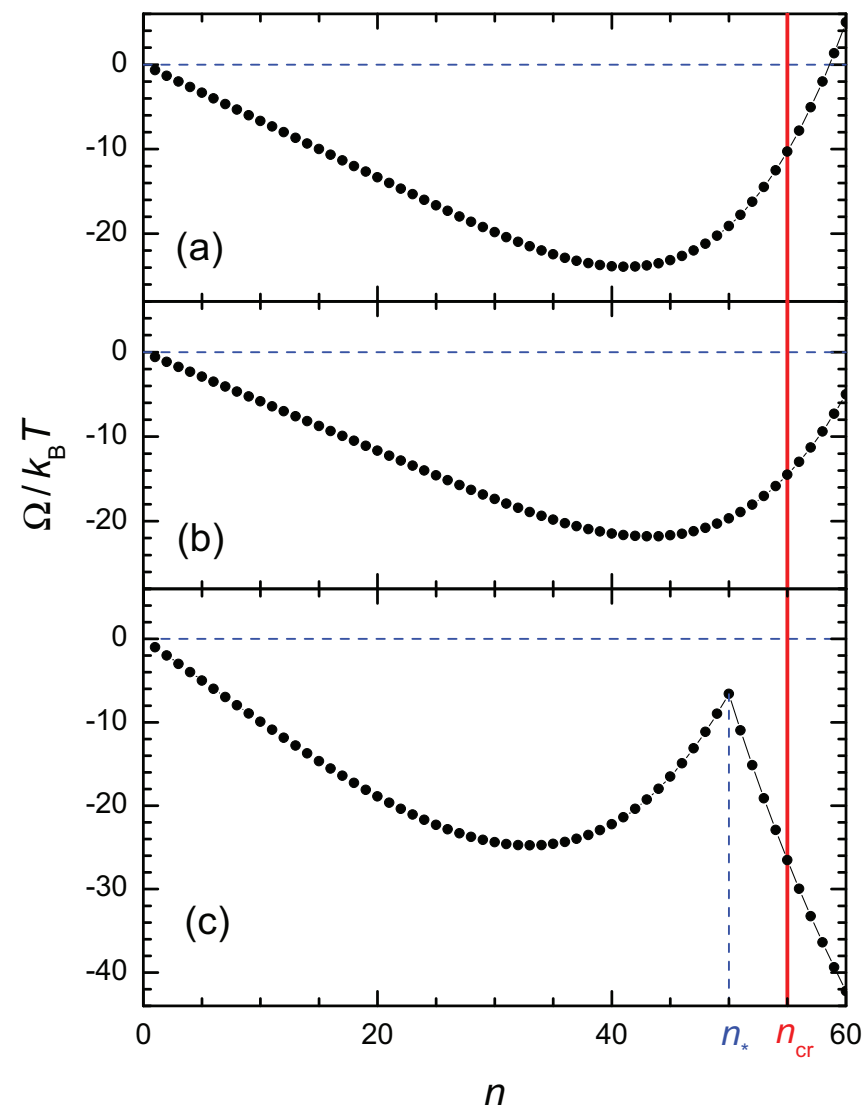

FIG. 3. (Color online) Qualitatively different dependencies of the grand potential on the number of ligand-receptor bonds, $n \leqslant m=$ 60, during virion engulfment: [(a) and (b)] the energy of cytoskeleton deformation increases up to $n=m$; (c) the energy of cytoskeleton deformation reaches maximum at $n=n_{*}<m$. Rupture or scission of the host-cell membrane is assumed to be possible at $n>n_{\mathrm{cr}}=55$. (These plots are not used for quantitative calculations below. For this reason, we do not specify the parameters employed to construct the figure. All the values are just to show their scale.)

$n=n_{*}$, and afterwards decreases with increasing $n$, i.e., the states with $n \geqslant n_{\text {cr }}$ becomes statistically more favorable with increasing $n$.

If the deformation energy of cytoskeleton is fully repulsive, the situation depends on whether $\Omega(m)$ is above [Fig. 3(a)] or below [Fig. 3(b)] zero (note that zero is associated with detachment). In the former case, the grand potential in the rupture or scission region (at $n \geqslant n_{\mathrm{cr}}$ ) may be appreciably or slightly [as shown in Fig. 3(a)] above zero. This means that the probability of membrane rupture or scission is, respectively, much lower than or comparable to that of virion detachment, because the barrier for detachment is lower than that for reaching the region where the scission may take place. In reality, this is usually not the case [2]. A more realistic situation is when $\Omega(m)$ is below zero [Fig. 3(b)].

If the deformation energy of cytoskeleton first increases and then decreases, the situation depends on whether $\Omega\left(n_{*}\right)$, i.e., the maximum of $\Omega(n)$, is above or below zero. In the former case, the probability of membrane scission is comparable with or lower than that of virion detachment. In the latter and more likely case [Fig. 3(c)], the membrane scission dominates.

\section{KINETICS}

\section{A. General kinetic equations}

The kinetics of endocytosis can be described in terms of the probabilities $p_{n}$ that a virion has $n(1 \leqslant n \leqslant m)$ ligand-receptor bonds. The process starts at $t=0$ by formation of the first bond, i.e., $p_{n}(0)=1$ and 0 for $n=1$ and $n>1$, respectively. At $t>0$, there is interplay of processes of formation and rupture of the ligand-receptor bonds with inclusion of rupture (or scission) of the membrane neck at the late stage. The corresponding kinetic equations for $p_{n}$ with $n=1,1<$ $n<n_{\mathrm{cr}}, n_{\mathrm{cr}} \leqslant n<m$, and $n=m$ are, respectively, as follows:

$$
\begin{gathered}
d p_{1} / d t=-k_{1} c p_{1}-\kappa_{1} p_{1}+\kappa_{2} p_{2}, \\
d p_{n} / d t=k_{n-1} c p_{i-1}-k_{n} c p_{n}-\kappa_{n} p_{n}+\kappa_{n+1} p_{n+1}, \\
d p_{n} / d t=k_{n-1} c p_{n-1}-k_{n} c p_{i}-\kappa_{n} p_{i}+\kappa_{n+1} p_{n+1}-r_{n} p_{n},
\end{gathered}
$$

$$
d p_{m} / d t=k_{m-1} c p_{m-1}-\kappa_{m} p_{m}-r_{m} p_{m},
$$

where $k_{n}, \kappa_{n}$ and $r_{n}$ are the rate constants of formation and rupture of the ligand-receptor bonds and of rupture or scission of the membrane neck, resulting in completion of the whole process. (In reality, the scission often occurs with participation of a special enzyme, e.g., Dynamin-2 [2]. Using the rate constants $r_{n}$, we do not describe explicitly this factor.)

The formation of new ligand-receptor bonds occurs primarily near the circular rim at the contact area. The activation energy of this step is expected to be low and its dependence on $n$ can be neglected. For these reasons, the corresponding rate constant can be represented as

$$
k_{n}=l(n) k_{\circ},
$$

where $k_{\circ}$ is the rate constant independent of $n$, and

$$
l(n)=2 \pi^{1 / 2}[n(1-n / m)]^{1 / 2}
$$

is the rim length calculated in the units characterizing the size of the contact area per receptor. [A similar approximation is widely used for the protein-attachment rate constant in the kinetic models of capsid assembly; see, e.g., [5](a,b,f).]

The detachment of receptors from a virion occurs at the rim as well, and accordingly the corresponding rate constant is also expected to be proportional to the rim length and can be represented as

$$
\kappa_{n}=l(n) \chi(n),
$$

where $\chi(n)$ is the factor taking into account that the detachment depends on the energetics of the interaction between a virion and the host cell. To specify $\chi(n)$, we notice that equilibrium between the formation and rupture of the bonds is described as

$$
l(n-1) k_{\circ} c p_{n-1}=l(n) \chi(n) p_{n} .
$$

On the other hand, the grand canonical distribution with potential (10) yields

$$
\begin{aligned}
\frac{p_{n}}{p_{n-1}} & =\exp \left(-\frac{\Omega(n)-\Omega(n-1)}{k_{\mathrm{B}} T}\right) \\
& =\exp \left(\frac{\epsilon+\mu-8 \pi \kappa / m-\Delta \mathcal{E}(n)}{k_{\mathrm{B}} T}\right),
\end{aligned}
$$


where

$$
\Delta \mathcal{E}(n)=B\left[f_{\mathrm{c}}(n)-f_{\mathrm{c}}(n-1)\right] .
$$

Taking into account that the ratio $p_{n} / p_{n-1}$ given by (18) and (19) should be identical and neglecting the difference between $l(n-1)$ and $l(n)$ in (18), we obtain

$$
\chi(n)=\left(k_{\circ} / a\right) \exp \left\{[-\epsilon+8 \pi \kappa / m+\Delta \mathcal{E}(n)] / k_{\mathrm{B}} T\right\} .
$$

Substituting this expression into (17), we have

$$
\kappa_{n}=l(n) \kappa_{\circ} \exp \left\{[-\epsilon+8 \pi \kappa / m+\Delta \mathcal{E}(n)] / k_{\mathrm{B}} T\right\},
$$

where $\kappa_{\circ} \equiv k_{\circ} / a$.

The rate constants of membrane rupture or scission $r_{n}$ also depend on $n$. At present, this dependence can hardly be accurately specified. The main point here is that this step happens on the late stage of the virion engulfment. For this reason, the details of the dependence of $r_{n}$ on $n$ are not crucial for the understanding of the kinetics of virus entry.

In addition to the steps described above, a virion can degrade. In principle, the latter process can be easily taken into account by including the corresponding terms into Eqs. (11)-(14). In reality, however, the acts of penetration of virions into cells are usually rather rapid. In the case of clathrin-mediated endocytosis, for example, it often takes a few minutes [2]. On this time scale, the virion degradation is expected to be negligible, and we can use Eqs. (11)-(14).

\section{B. Analytical results}

As already noted, endocytosis can be viewed as potentialdriven diffusion in the space of $n$. Taking the shape of the grand potential (Fig. 3) into account, the whole process can be divided into two phases. The first phase occurring just after virion attachment (with $n=1$ ) to the host cell (or after reaching a favorable region for engulfment during migration along the host-cell membrane) represents downhill diffusion towards the minimum of the grand potential. This very short phase is controlled primarily by transitions from $n$ to $n+1$. The time interval characterizing each transition is $1 / k_{n} c$, and accordingly the duration of the whole phase can be estimated as

$$
\tau_{1} \simeq \int_{n=1}^{n_{\star}} \frac{d n}{k_{n} c},
$$

where $n_{\star}$ is the number corresponding to the minimum of the grand potential. Substituting (15) in combination with (16) into (23) then yields

$$
\tau_{1} \simeq \frac{m^{1 / 2} \arcsin \left(n_{\star} / m\right)}{\pi^{1 / 2} k_{\circ} c} .
$$

After reaching the bottom of the grand potential, the increase and decrease of $n$ is statistically not favorable, and the rates of transitions $n \rightarrow n+1$ and $n+1 \rightarrow n$ are much faster than the resulting rates of membrane penetration and detachment. This situation is qualitatively similar to that occurring in conventional chemical reactions. This means that there is a long steady-state phase ending either by membrane penetration by a virion or by its detachment. During this important phase, endocytosis can be described as a first-order process with a well-defined rate constant. In particular, the corresponding rate constant $\gamma$ can be calculated by solving Eqs. (11)-(14) in the steady-state approximation. Mathematically, Eqs. (11)-(14) are similar to those widely used to describe chemical reactions, and the ways it can be done are well known since the seminal article by Kramers [28] (see, e.g., compilation in [29]). The final expressions for $\gamma$ are typically cumbersome and depend on various details.

In our context, as already noticed in Sec. II B, we are interested in two qualitatively different situations [Figs. 3(b) and 3(c)] when the detachment probability is relatively low. If the grand potential depends on $n$ as shown in Fig. 3(b), there is an interplay at $n>n_{\mathrm{cr}}$ between bond formation (activation) and membrane rupture (reaction). Due to this interplay, there exists a rate determining number of bonds $n_{\bullet}$, and the virus entry rate constant can approximately be represented as

$$
\gamma \simeq r_{n_{\bullet}} \exp \left\{-\left[\Omega\left(n_{\bullet}\right)-\Omega\left(n_{\star}\right)\right] / k_{\mathrm{B}} T\right\} .
$$

If the grand potential behaves as shown in Fig. 3(c), there is a well-defined potential barrier at $n=n_{*}$ for endocytosis, and the entry rate constant is approximately given by

$$
\gamma \simeq k_{n_{*}-1} \exp \left\{-\left[\Omega\left(n_{*}-1\right)-\Omega\left(n_{\star}\right)\right] / k_{\mathrm{B}} T\right\} .
$$

In both cases, the time scale of the second phase of the entry is determined as

$$
\tau_{2}=1 / \gamma
$$

In fact, this time scale characterizes the whole process, because the first phase is negligibly short.

\section{Results of calculations}

To complement the analytical results given in the previous subsection and to illustrate the predictions of the model more explicitly, we have calculated the kinetics of virus entry in the case when the grand potential (10) has a maximum as shown in Fig. 4(a). The parameters used to construct the potential were $m=60, n_{\circ}=10, n_{*}=50, \epsilon-8 \pi \kappa / m=3 k_{\mathrm{B}} T, B=$ $10^{-5} k_{\mathrm{B}} T$, and $c a=0.1$. Note that the chosen value of $m$ is typical for many viruses. The values of $n_{\circ}=10$ and $n_{*}=50$ are reasonable for the typical structures of filament networks (see, e.g., [24]). The energetic parameters, $\epsilon-8 \pi \kappa / m$ and $B$, are the most important. The former one depends primarily on the binding energy per receptor. This energy is often mentioned in the original experimental and theoretical studies and reviews (see, e.g., theoretical treatments [12-14,17] and references used there to validate the parameters) but its accurate measurements are still rare. For orientation, we mention that $\epsilon$ may be up to $10 k_{\mathrm{B}} T$ [17](b) (if $c$ is low, a receptor can be efficient only provided $\epsilon$ is relatively large). The value we use, $\epsilon-8 \pi \kappa / m=3 k_{\mathrm{B}} T$, is comparable to that estimated for glycosphingolipids [10](c) (concerning these lipids, see also [30]). The value of $B$ depends on the structure and elastic constants of actin filaments (for the corresponding data, see, e.g., [24,26]). The value we employ, $B=10^{-5} k_{\mathrm{B}} T$, is in line with the earlier estimates [18]. Concerning the receptor concentration, we used $c a=0.1$. [In fact, the results of our calculations presented below depend on the combination of $\epsilon-8 \pi \kappa$ and $c a$ or, more specifically, on $\epsilon-8 \pi \kappa+\ln (c a)$. If one employs $c a=0.01$, the results will be the same provided $\epsilon-8 \pi \kappa / m=5.3 k_{\mathrm{B}} T$.] 

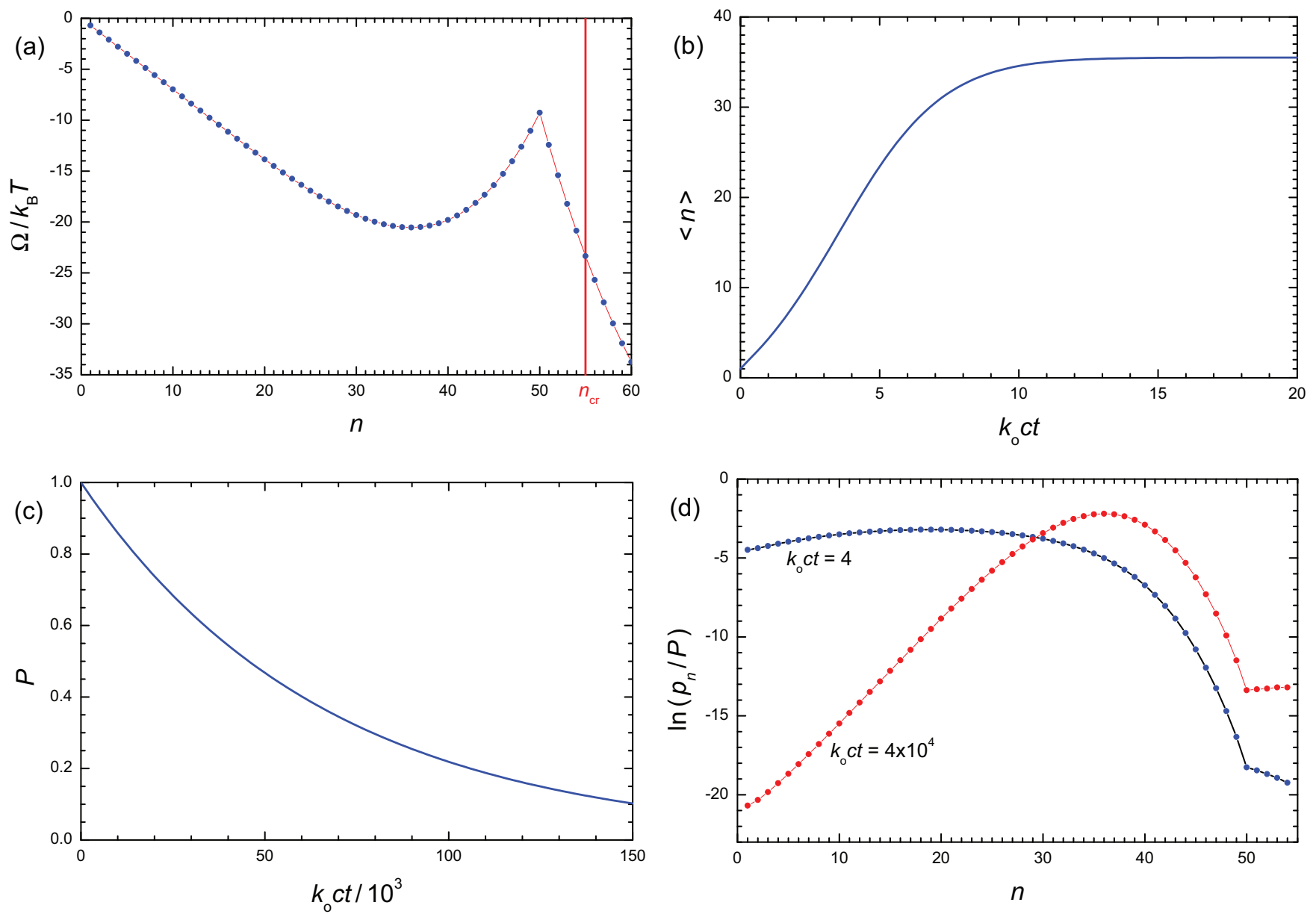

FIG. 4. (Color online) Calculations illustrating the kinetics of virus entry: (a) grand potential (10) as a function of the number of the ligand-receptor bonds (for the corresponding values of the parameters, see the text); (b) average value of $n,\langle n\rangle=\sum_{n} n p_{n} / \sum_{n} p_{n}$, as a function of time in the very beginning of the process; (c) probability of finding a virion at the membrane, $P=\sum_{n} p_{n}$; and (d) logarithm of the normalized probabilities, $\ln \left(p_{n} / P\right.$ ), for the first and second phases of the kinetics at $k_{\circ} c t=4$ and $4 \times 10^{4}$, respectively (during the second phase, the distribution of these probabilities is close to the equilibrium one and nearly independent of time).

With the parameters above, the entry rate is limited by crossing the potential barrier at $n=n_{*}$, and the details of the mechanism of membrane rupture at $n \geqslant n_{\mathrm{cr}}$ are of minor importance. In fact, the steps at $n \geqslant n_{\text {cr }}$ can be replaced by the absorbing boundary condition at $n=n_{\mathrm{cr}}$, i.e., $p_{n_{\mathrm{cr}}}=0$. To reduce the number of parameters, the virion detachment was neglected by setting $\kappa_{1}=0$. With this specification, Eqs. (11)-(14) for $p_{n}$ with $1 \leqslant n \leqslant n_{\text {cr }}-1$ were integrated numerically as a function of $k_{\circ} c t$.

The results of calculations [Figs. 4(b)-4(d)] are in agreement with the analysis in the preceding subsection. In particular, Fig. 4(b) shows the average value of $n$,

$$
\langle n\rangle=\sum_{n} n p_{n} / \sum_{n} p_{n},
$$

as a function of time. With increasing time, one can observe a rapid transition from the first phase with downhill diffusion in the space of $n$ to the steady-state regime with $\langle n\rangle$ close to that corresponding to the minimum of $\Omega$. The time scale characterizing the duration of the first stage, $\tau_{1} \simeq 5 / k_{\circ} c$, is in agreement with that predicted by Eq. (24). The virus entry occurs during the second steady-state phase at $t \gg \tau_{1}$, and the corresponding kinetics is exponential [Fig. 4(c)]. Note that $\langle n\rangle$ is calculated for remaining virions, and accordingly it is constant under steady-state conditions [Fig. 4(b)] despite the exponential decrease of the probability to find a virion at the membrane [Fig. 4(c)]. Typical distributions of $p_{n}$ at these two stages [Fig. 4(d)] are, as expected, far and close to the equilibrium one, respectively.

\section{CONCLUSION}

The analytical and numerical results of our study of the kinetics of virus entry by endocytosis can be summarized as follows. The process is governed by the dependence of the grand potential on the number of ligand-receptor bonds which in turn is crucially dependent on deformation of actin filaments forming the cytoskeleton of the host cell [Eq. (10)]. It can be divided into a rapid initial stage and a long steady-state stage. The entry occurs during the latter stage and can be described as a first-order reaction. Depending on the details of the behavior of the grand potential, the process can be limited either by the interplay of reversible bond formation and membrane rupture (or scission) or by reaching a maximum of this potential. 
Finally, we note that some of the ingredients of our analysis may be applicable also to the treatments of the fusogenic mechanism of virus entry, because it is also related to the formation of the ligand-receptor bonds, membrane and actin-filament deformation, and subsequent membrane rupture. In analogy with endocytosis, the virus entry via this channel is expected to occur during the steady-state stage and also can be described as a first-order reaction.

\section{ACKNOWLEDGMENTS}

This work was supported by Swedish Research Council. The author thanks F. Höök for useful discussions.
[1] A. J. Cann, Principles of Molecular Virology (Elsevier, Amsterdam, 2012).

[2] J. Mercer, M. Schelhaas, and A. Helenius, Ann. Rev. Biochem. 79, 803 (2010).

[3] Y. Sidorenko, A. Voigt, J. Schulze-Horsel, U. Reichl, and A. Kienle, Chem. Eng. Sci. 63, 2299 (2008); J. Sardanyes and S. F. Elena, PLoS ONE 6, e24884 (2011).

[4] M. Bally, K. Dimitrievski, G. Larson, V. P. Zhdanov, and F. Höök, Phys. Biol. 9, 026011 (2012).

[5] R. Zandi, P. van der Schoot, D. Reguera, W. Kege, and H. Reiss, Biophys. J. 90, 1939 (2006); A. Yu. Morozov, R. F. Bruinsma, and J. Rudnick, J. Chem. Phys. 131, 155101 (2009); A. Zlotnick, J. Z. Porterfield, and J. C.-Y. Wang, Biophys. J. 104, 1595 (2013); M. Castelnovo, T. Verdier, and L. Foret, Eur. Phys. Lett. 105, 28006 (2014); E. C. Dykeman, P. G. Stockley, and R. Twarock, Proc. Natl. Acad. Sci. USA 111, 5361 (2014); V. P. Zhdanov, Phys. Rev. E 90, 042721 (2014).

[6] M. G. Mateu, Arch. Biochem. Biophys. 531, 65 (2013).

[7] E. Barrow, A. V. Nicola, and J. Liu, Virology J. 10, 177 (2013).

[8] Y. Yamauchi and A. Helenius, J. Cell. Sci. 126, 1289 (2013).

[9] I. Canton and G. Battaglia, Chem. Soc. Rev. 41, 2718 (2012).

[10] H. M. van der Schaar, M. J. Rust, C. Chen, H. van der Ende-Metselaar, J. Wilschut, X. Zhuang, and J. M. Smit, PLoS Path. 4, e1000244 (2008); D. Sivaraman, P. Biswas, L. N. Cella, M. V. Yates, and W. Chen, Trends Biotechnol. 29, 307 (2011); M. Bally, A. Gunnarsson, L. Svensson, G. Larson, V. P. Zhdanov, and F. Höök, Phys. Rev. Lett. 107, 188103 (2011).

[11] A. Handel, V. Akin, S. S. Pilyugin, V. Zarnitsyna, and R. Antia, J. R. Soc. Interf. 11, 20131083 (2014).

[12] T. J. English and D. A. Hammer, Biophys. J. 86, 3359 (2004); 88, 1666 (2005); T. Chou and M. R. D’Orsogna, J. Chem. Phys. 127, 105101 (2007); O. M. Szklarczyk, N. GonzalezSegredo, P. Kukura, A. Oppenheim, D. Choquet, V. Sandoghdar, A. Helenius, I. F. Sbalzarini, and H. Ewers, PLoS Comput. Biol. 9, e1003310 (2013).

[13] T. Chou, Biophys. J. 93, 1116 (2007); S. A. Nowak and T. Chou, ibid. 96, 2624 (2009).

[14] M. M. Gibbons, T. Chou, and M. R. D’Orsogna, J. Phys. Chem. B 114, 15403 (2010).

[15] S. A. Nowak and T. Chou, Phys. Rev. E 78, 021908 (2008).
[16] V. P. Zhdanov, Cent. Eur. J. Phys. 10, 1210 (2012).

[17] S. X. Sun and D. Wirtz, Biophys. J. 90, L10 (2006); A. Gefen, J. Biomech. Eng. 132, 084501 (2010); L. Li, X. Liu, Y. Zhou, and J. Wang, Biophys. J. 102, 2230 (2012).

[18] V. P. Zhdanov, Phys. Rev. E 88, 064701 (2013).

[19] P. Decuzzi and M. Ferrari, Biophys. J. 94, 3790 (2008); R. Vacha, F. J. Martinez-Veracoechea, and D. Frenkel, Nano Lett. 11, 5391 (2011); S. Dasgupta, T. Auth, and G. Gompper, Soft Matter 9, 5473 (2013); M. Raatz, R. Lipowsky, and T. R. Weikl, ibid. 10, 3570 (2014); A. H. Bahrami, M. Raatz, J. Agudo-Canalejo, R. Michel, E. M. Curtis, C. K. Hall, M. Gradzielski, R. Lipowsky, and T. R. Weikl, Adv. Coll. Interf. Sci. 208, 214 (2014).

[20] V. P. Zhdanov, Elementary Physicochemical Processes on Solid Surfaces (Plenum, New York, 1991).

[21] J. A. Jackman, G. H. Zan, V. P. Zhdanov, and N.-J. Cho, J. Phys. Chem. B 117, 16117 (2013).

[22] V. P. Zhdanov and B. Kasemo, Langmuir 17, 3518 (2001).

[23] B. Antonny, Ann. Rev. Biochem. 80, 101 (2011); T. Baumgart, B. R. Capraro, C. Zhu, and S. L. Das, Annu. Rev. Phys. Chem. 62, 483 (2011); V. P. Zhdanov and F. Höök, Biophys. Chem. 170, 17 (2012); A. Tonnesen, S. M. Christensen, V. Tkach, and D. Stamou, Biophys. J. 106, 201 (2014); M. Rabe, S. R. Tabaei, H. Zetterberg, V. P. Zhdanov, and F. Höök, Angew. Chem. Int. Ed. 54, 1022 (2015).

[24] F. Huber, J. Schnaus, S. Rönicke, P. Rauch, K. Müller, C. Fütterer, and J. Käs, Advances Phys. 62, 1 (2013).

[25] M. F. Coughlin and D. Stamenović, Biophys. J. 84, 1328 (2003); D. A. Head, A. J. Levine, and F. C. MacKintosh, Phys. Rev. E. 68, 061907 (2003); S. Roy and H. J. Qi, Phys. Rev. E 77, 061916 (2008); X. M. Mao, O. Stenull, and T. C. Lubensky, Phys. Rev. E. 87, 042602 (2013).

[26] T.-J. Chen, C.-C. Wu, and F.-C. Su, Med. Engin. Phys. 34, 1375 (2012).

[27] M. Dhamala, V. K. Jirsa, and M. Ding, Phys. Rev. Lett. 92, 028101 (2004)

[28] H. A. Kramers, Physica 7, 284 (1940).

[29] E. E. Nikitin, Theory of Elementary Atomic and Molecular Processes in Gases (Clarendon, Oxford, 1974); P. Hänggi, P. Talkner, and M. Borkovec, Rev. Mod. Phys. 62, 251 (1990).

[30] M. E. Breimer, G. C. Hansson, K.-A. Karlsson, G. Larson, and H. Leffler, Glycobiol. 22, 1721 (2012). 